\title{
Serum biochemical and inflammatory cytokines in a rat model of metabolic syndrome
}

\author{
Mohammed Haruna Yeldu', Ahmed Mus'ab² \\ ${ }^{1}$ Associate Professor, Department of Chemical Pathology, School of Medical Laboratory Sciences, Usmanu Danfodiyo \\ University Sokoto, Nigeria, ${ }^{2}$ Medical Laboratory Scientist, Department of Immunology, School of Medical Laboratory \\ Sciences, Usmanu Danfodiyo University Sokoto, Nigeria
}

A B S T R A C T

Background: Metabolic syndrome (MetS) is a combination of cardio-metabolic risk factors including obesity, hyperglycaemia, dyslipidaemia, oxidative stress and hypertension. Aims and Objectives: This study was aimed at determining the serum concentrations of biochemical and inflammatory cytokines and assesses the correlation between the biochemical and inflammatory cytokines in a rat model of metabolic syndrome. Materials and Methods: Twenty rats were divided into 2 groups of 10 each: control (fructose untreated) and the fructose treated group. MetS was induced by oral administration of $10 \%$ fructose in water for 32 weeks. At the end of the experiment, rats were fasted for 12 hours and blood samples collected and body weight, body mass index (BMI), fasting blood glucose (FBG), serum lipid profile, interleukin-6 (IL-6), interleukin-10 (IL-10), tumour necrosis factor- $\alpha$ (TNF- $\alpha$ ) were measured using standard techniques. Result: Result indicated significantly $(P<0.05)$ increased FBG, body weight, BMI, serum TC and LDL-C while, HDL-C levels significantly $(P<0.05)$ decreased in MetS group compared with controls. However, the levels of VLDL-C, TG and AIX were not significantly $(P>0.05)$ different between the groups. Significantly $(P<0.05)$ increased serum IL-6, IL-10 and TNF- $\alpha$ were observed in MetS group compared with controls. Serum IL-10 and TNF- $\alpha$ were inversely correlated with BMI. Serum IL-10 negatively correlated with FBG while, IL-6 was not correlated with either of BMI and FBG.Serum AIX and VLDL-C were positively correlated with TG while, HDL-C level was inversely correlated with TG. With the exception of serum LDL-C which positively correlated with TC, significant correlation was not established between serum TC and each of AIX, HDL-C and VLDL-C. Conclusion: The rat model of MetS was established after treatment with $10 \%$ fructose in water. This plays an important role in the pathogenesis of components of metabolic syndrome, including dyslipidaemia, hyperglycaemia and obesity; and serum IL-6, IL-10 and TNF- $\alpha$ are raised in metabolic syndrome and this underscores the role of these cytokines in inflammation associated with metabolic syndrome.

Key words: Metabolic syndrome; Biochemical and Inflammatory cytokines; Wistar rats

\section{Access this article online}

Website:

http://nepjol.info/index.php/AJMS DOI: 10.3126/ajms.v11i4.28963

E-ISSN: 2091-0576

P-ISSN: $2467-9100$

Copyright (c) 2020 Asian Journal of Medical Sciences

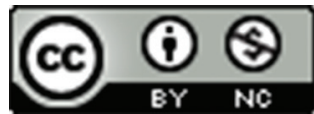

This work is licensed under a Creative Commons Attribution-NonCommercial 4.0 International License.

\section{INTRODUCTION}

Metabolic syndrome (MetS) is caused by the clustering of risk factors of insulin resistance (IR), hypertension, dyslipidaemia, central obesity, pro-inflammatory and prothrombotic states which leads to substantially increased morbidity of cardiovascular diseases (CVD) and diabetes mellitus type 2 (T2DM). ${ }^{1-2}$
The clustering of these risk factors and their relationship to lifestyle changes suggests that they are dependent on one another and have similar underlying causes, mechanisms and characteristics. ${ }^{3,4}$

Patients with the MetS have altered blood cytokines and growth factor profile that may partially account for the adverse clinical outcomes seen in MetS. ${ }^{5}$ There is an up- 
regulation of certain pro- and anti-inflammatory cytokines in obesity; general obesity is associated with significantly elevated levels of interleukin (IL)-5, IL-10, IL-12, IL-13, interferon (IFN- $\gamma$ ) and tumour necrosis factor (TNF- $\alpha$ ), while significantly increased levels of IL-5, IL-10, IL-12, IL-13 and IFN- $\gamma$-were observed in central obesity. ${ }^{6}$

Leading factors for the development of the symptom complex include obesity, reduced physical activity and improper diet. ${ }^{7}$ Components of the syndrome are risk factors for atherosclerosis, making metabolic syndrome a significant risk for coronary heart disease. ${ }^{8}$ Obesity and IR also provide significant risk for developing type 2 diabetes mellitus. ${ }^{9}$

Over recent decades, fructose consumption has increased dramatically in industrialized countries as manufacturers have turned to using fructose instead of sucrose to sweeten processed foods and beverages. Similarly, the intake of saturated fat has risen during the same period. ${ }^{10}$ High dietary intake of fructose and saturated fat contributes to the epidemic of the metabolic syndrome, ${ }^{8}$ which was generally considered to be an association of impaired glucose tolerance, hypertension, dyslipidaemia, hyperuricaemia, and central obesity. ${ }^{9}$

Metabolic syndrome is increasing, in accordance with increasing body mass index (BMI) and age. ${ }^{11}$ Worldwide prevalence of MetS was reported to be between 10\% and $84 \%$, and this depends on the ethnicity, age, gender and race of the population. ${ }^{12}$ About $20 \%$ of adults in the Western world have also been reported to have MetS. ${ }^{13}$

Several approaches have been used to measure risk factors of MetS. This study was aimed at determining the serum concentrations of biochemical and inflammatory cytokines in a rat model of metabolic syndrome. This may provide information that may aid in the prevention and management of biochemical derangements and inflammation associated with metabolic syndrome.

\section{MATERIALS AND METHODS}

\section{Study area}

The study was carried out in the Departments of Chemical Pathology and Immunology, School of Medical Laboratory Sciences, Usmanu Danfodiyo University, Sokoto, and Animal House, Faculty of Pharmaceutical Sciences, Usmanu Danfodiyo University, Sokoto.

\section{Reagents/Chemicals}

Analytical graded chemicals and reagents were used for this research. ELISA test kits for serum IL-6, IL-10 and TNF- $\alpha$ assay were obtained from Sigma-Aldrich ${ }^{\circledR}$, Saint Louis, MO,
USA. Serum lipid profile and plasma glucose assay kits were procured from Randox Laboratories England.

\section{Experimental animals}

Twenty (20) male Wistar Albino rats weighing between 100-140 g were purchased from Faculty of Pharmaceutical Sciences, Usmanu Danfodiyo University Sokoto. The rats were housed in well aerated cages under hygienic conditions and allowed to acclimatize for a period of two weeks before commencement of the experiment. The animals were fed with pelletized growers feed $\left(\mathrm{Vital}^{\circledR}\right)$, obtained from Grand Cereal Soil Mills Limited, Jos, Nigeria. They were also allowed access to clean drinking water ad libitum throughout the experimental period. Cleaning of the animal cages was carried out daily, and on regular basis. They were maintained in clean metabolic cage-sand, placed in a well-ventilated room conditions with a temperature of $26^{\circ} \mathrm{C}$ to $28^{\circ} \mathrm{C}$, photoperiods of 12 hours light and 12 hours darkness; humidity of $40 \%$ to $60 \%$ as described by the National Research Council (US) Committee for the Update of the Guide for the Care and Use of Laboratory Animals in 2011. ${ }^{14}$

\section{Ethical consideration}

All the experimental protocols were in compliance with the Animal Ethics Committee of Usmanu Danfodiyo University, Sokoto, Nigeria, as well as internationally accepted practices for use and care of laboratory animals as contained in U.S.A. guidelines, and also in accordance with the recommendations of the International Association for the study of Pain.

\section{Experimental design}

Twenty (20) rats were randomly divided into two groups of 10 rats each. Control group $(n=10)$ : untreated/ on drinking water and standard rodent chow ad-libitum for 32 weeks. MetS group $(\mathrm{n}=10)$ : Fructose-loaded / treated with $10 \%$ fructose in drinking water (w/v) and standard rodent chow ad-libitum for 32 weeks. ${ }^{15}$

\section{Anthropometric measurements}

The body weights of all rats were measured using a sensitive weighing balance, once before the commencement of fructose dosing and once on the day of sacrifice (32 weeks). The weight in gram (g) of each rat was measured by placing the rat into a container placed on a sensitive weighing balance after zeroing the machine. ${ }^{16}$

The body length of each rat was measured from anus to the tip of the nose in centimetres $(\mathrm{cm})$ once before the commencement of fructose dosing and once on the day of sacrifice. The body mass index (BMI) of each rat was calculated using the following expression:BMI $\left(\mathrm{g} / \mathrm{cm}^{2}\right)=$ body weight (gram)/ body length $\left(\mathrm{cm}^{2}\right)$. 
Blood sample collection and processing

After 32 weeks of the experiment, the animals were fasted for 12 hours, and were anaesthetized in a glass jar containing wool soaked with chloroform. About five millilitres (5ml) of blood samples were collected from each animal through cardiac puncture. Four (4) millilitres of the blood samples were collected into clean, plain Vacutainer tubes. The samples collected were allowed to clot at room temperature and later centrifuged at 4000 revolution per minute (4000 $\mathrm{rpm}$ ) for 10 minutes. The sera were then transferred into labelled sterile serum bottles and tightly caped and stored at $-20{ }^{\circ} \mathrm{C}$ until used for the measurement of serum IL-6, IL-10, TNF- $\alpha$, and biochemical parameters. The remaining 1 millitre of blood was collected into fluoride oxalate bottle for estimation of fasting plasma glucose within one hour of the sample collection. Humane procedures were used in the process.

\section{Analytical methods}

Serum total cholesterol (TC), triglyceride (TG), high density lipoprotein cholesterol (HDL-C), low density lipoprotein cholesterol (LDL-C), very low density lipoprotein cholesterol (VLDL-C) and plasma glucose, were estimated using test kits procured from Randox Laboratories, England. Atherogenic Index was calculated as the ratio of TC to HDL-C. ${ }^{17}$ Serum IL-6, IL-10 and TNF- $\alpha$ were estimated by the method of Yener et al. ${ }^{18}$ using ELISA kits obtained from Sigma-Aldrich $®$, Saint Louis, MO, USA.

\section{Statistical analysis}

Data was analysed using GraphPad Prism (GraphPad Software, 2365 Northside Dr. Suite 560 San Diego, CA 92108). Values were expressed as mean \pm standard error of mean for rats in each group. The data of anthropometric, biochemical and immunological parameters were analysed using independent sample t-test. Pearson correlation coefficient was used to assess the relationship between the parameters analysed, while the differences were considered significant when $P$ value is equal to or less than 0.05 $(\mathrm{p} \leq 0.05)$.

\section{RESULTS}

Chronic administration of fructose as a $10 \%$ drinking solution for 32 weeks induced several alterations included in the cluster of risk factors of MetS. At the end of the experiment, the fasting blood glucose concentrations, final body weight and body mass index of fructose-induced metabolic syndrome (MetS) rats and controls (Table 1) indicated significantly increased FBG $(P<0.000)$, body weight $(P<0.000)$ and BMI $(P<0.000)$ in MetS group compared with control group $(P=0.912, P=0.455$ and $P=0.606$ respectively).

\begin{tabular}{|c|c|c|}
\hline Parameter & Controls $(n=10)$ & MetS $(n=10)$ \\
\hline \multicolumn{3}{|c|}{ FBG (mmol/L) } \\
\hline Week 1 & $3.16 \pm 0.18$ & $3.18 \pm 0.12$ \\
\hline Week 32 & $3.46 \pm 0.24$ & $9.30 \pm 0.80^{\alpha}$ \\
\hline$P$. values & 0.912 & $<0.000$ \\
\hline \multicolumn{3}{|c|}{ Body weight (g) } \\
\hline Week 1 & $205.40 \pm 1.31$ & $207.0 \pm 1.63$ \\
\hline Week 32 & $223.20 \pm 0.10$ & $272.30 \pm 2.28^{\alpha}$ \\
\hline$P$. values & 0.455 & $<0.000$ \\
\hline \multicolumn{3}{|l|}{ BMI $\left(\mathrm{g} / \mathrm{cm}^{2}\right)$} \\
\hline Week 1 & $0.53 \pm 0.03$ & $0.56 \pm 0.04$ \\
\hline Week 32 & $0.48 \pm 0.02$ & $0.73 \pm 0.05^{\alpha}$ \\
\hline$P$. values & 0.606 & $<0.001$ \\
\hline
\end{tabular}

Regarding the levels of serum lipids of MetS group compared to controls (Table 2$)$, serum TC $(P<0.000)$ and LDL-C $(P<0.000)$ were significantly increased. On the other hand, HDL-C levels were significantly decreased in MetS group $(P=0.040)$, while the levels of VLDL-C, TG and AIX were not significantly $(P=0.343$ and $P=0.214$ respectively) different between the groups. The serum levels of inflammatory cytokines in fructose-induced metabolic syndrome rats and controls is shown in Table 3. The result indicated significantly increased serum IL-6 $(P=0.005)$, IL-10 $(P<0.000)$ and TNF- $\alpha(P=0.013)$ in MetS group as compared with controls.

The correlation between each of serum IL-6, IL-10 and TNF- $\alpha$, with body mass index and blood glucose concentration of fructose-induced MetS group (Table 4) revealed that serum IL-10 and TNF- $\alpha$ were inversely correlated with BMI $(\mathrm{r}=-0.732, P=0.016 ; \mathrm{r}=-0.785$, $P=0.007$ respectively). Serum IL-10 also showed the same relationship with FBG $(r=-0.817, P=0.004)$ while, serum TNF- $\alpha$ was not associated with FBG $(r=0.205$, $P=0.570)$. Likewise serum IL-6 levels were not associated with either of BMI and FBG ( $\mathrm{r}=0.516, P=0.126$; $\mathrm{r}=0.205, P=0.570$ respectively) (Table 4 ).

The result of the correlation between each of serum TC and TG with serum AIX, HDL-C, LDL-C and VLDL-C of fructose-induced metabolic syndrome rats (Table 5) indicated that serum AIX and VLDL-C were positively correlated with TG $(\mathrm{r}=0.733, P=0.016 ; \mathrm{r}=1.000$, $P=0.000$ respectively) while, HDL-C level was inversely correlated with TG $(\mathrm{r}=-0.817, P=0.004)$.

With the exception of serum LDL-C which positively correlated with TC $(\mathrm{r}=0.840, P=0.002)$, significant correlation was not established between serum TC and each of AIX, HDL-C and VLDL-C ( $\mathrm{r}=0.588, P=0.016$; $\mathrm{r}=-0.210, P=0.560$ and $\mathrm{r}=0.262, P=0.464$ respectively). 
Table 2: Serum lipid profile and atherogenic index of fructose-induced MetS rats and controls

\begin{tabular}{lccccccc} 
Group & $\mathbf{N}$ & TC $(\mathbf{m m o l} / \mathbf{L})$ & HDL-C $(\mathbf{m m o l} / \mathbf{L})$ & LDL-C $(\mathrm{mmol} / \mathrm{L})$ & VLDL-C $(\mathrm{mmol} / \mathrm{L})$ & TG $(\mathrm{mmol} / \mathrm{L})$ & AIX \\
\hline Controls & 10 & $0.82 \pm 0.04$ & $0.33 \pm 0.04$ & $0.18 \pm 0.06$ & $0.41 \pm 0.03$ & $0.90 \pm 0.06$ & $4.26 \pm 0.56$ \\
MetS & 10 & $1.71 \pm 0.10^{\mathrm{b}}$ & $0.22 \pm 0.03^{\mathrm{a}}$ & $0.96 \pm 0.08^{\mathrm{b}}$ & $0.44 \pm 0.03$ & $0.98 \pm 0.06$ & $6.51 \pm 1.65$ \\
P. values & & $<0.000$ & 0.040 & $<0.000$ & 0.343 & 0.343 & 0.214 \\
\hline
\end{tabular}

Data expressed as mean $\pm \mathrm{SEM}, \mathrm{N}=$ number of rats, $\mathrm{TC}=$ =total cholesterol, $\mathrm{HDL}-\mathrm{C}=$ high density lipoprotein cholesterol, $\mathrm{LDL}-\mathrm{C}=$ low density lipoprotein cholesterol, $\mathrm{VLDL}-\mathrm{C}=\mathrm{very}$ low density lipoprotein cholesterol, TG=triglyceride, $\mathrm{AIX}=$ atherogenic index, MetS=metabolic syndrome rats, ${ }^{\mathrm{a}} \mathrm{P}<0.05$, ${ }^{\mathrm{b}} \mathrm{P}<0.001$ when compared with control rats using Student t-test

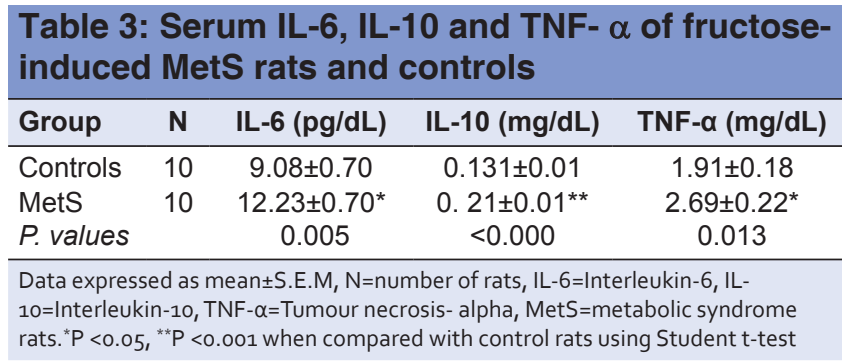

\begin{tabular}{|c|c|c|c|c|}
\hline \multirow[t]{2}{*}{ Parameter } & \multicolumn{2}{|c|}{ BMI $\left(\mathrm{g} / \mathrm{cm}^{2}\right)$} & \multicolumn{2}{|c|}{ FPG (mmol/L) } \\
\hline & $\mathbf{r}$ & $P$ & $r$ & $P$ \\
\hline IL-6 (pg/dl) & 0.516 & 0.126 & -0.250 & 0.486 \\
\hline IL-10 (mg/dl) & -0.732 & 0.016 & -0.817 & 0.004 \\
\hline TNF- $\alpha(\mathrm{mg} / \mathrm{dL})$ & -0.785 & 0.007 & 0.205 & 0.570 \\
\hline
\end{tabular}

Data expressed as correlation coefficient $(r), P=P=$ value, $\mathrm{BMI}=$ body mass index, FPG=fasting plasma glucose, IL-6=Interleukin- $6, \mathrm{IL}-10=$ Interleukin-10, TNF- $\alpha=$ Tumour necrosis- alpha, MetS=metabolic syndrome rats

\section{DISCUSSION}

In the current study, the effect of $10 \%$ fructose in drinking water, which is equivalent to a diet containing 48-57\% by calories, was examined on metabolic characteristics in Wistar rats. The results in this study, indicated that fructose enriched diet could induce dyslipidaemia, increase fasting blood glucose concentration, body weight and body mass index in fasting rats.

The result of this study corroborated with previous study. ${ }^{15}$ The significant increase in fasting blood glucose (FBG) observed in MetS rats is in agreement with several studies. ${ }^{15,19-22}$ However, our result contrasted with the previous studies ${ }^{22,23}$ that observed no changes in the FBG levels between MetS and control groups. Sobczak et al. ${ }^{24}$ in their study reported that, the significant elevation of blood glucose levels may be the result of accumulation of non-esterified free fatty acids which may reduce insulin sensitivity by increasing the intra myo-cellular lipid content. Whereas the discrepancy observed in blood glucose could be due to the differences in the analytical methods used in

\begin{tabular}{|c|c|c|c|c|}
\hline \multirow[t]{2}{*}{ Parameter } & \multicolumn{2}{|c|}{ TC (mmol/L) } & \multicolumn{2}{|c|}{ TG (mmol/L) } \\
\hline & $r$ & $P$ & $r$ & $P$ \\
\hline AIX & 0.588 & 0.074 & 0.733 & 0.016 \\
\hline $\mathrm{HDL}-\mathrm{C}(\mathrm{mmol} / \mathrm{L})$ & -0.210 & 0.560 & -0.817 & 0.004 \\
\hline LDL-C (mmol/L) & 0.840 & 0.002 & 0.189 & 0.600 \\
\hline VLDL-C (mmol/L) & 0.262 & 0.464 & 1.000 & 0.000 \\
\hline
\end{tabular}

Data expressed as correlation coefficient $(r), P=P=$ value, $T C=$ total cholesterol, TG=triglyceride, $\mathrm{HDL}=$ high density lipoprotein cholesterol, $\mathrm{LDL}-\mathrm{C}=$ low density lipoprotein cholesterol, VLDL-C=very low density lipoprotein cholesterol, $\mathrm{AIX}=$ atherogenic index

the estimation of glucose. In this study, glucose oxidaseperoxidase specific method was used while, Mohammed et $a .^{22}$ and Norshalizah et al. ${ }^{23}$ both used the chemical methods (Folin Wu) for glucose estimation.

Administration of fructose in drinking water significantly increased body weight and BMI in MetS rats compared with the controls. This is in agreement with the study of Norshalizah et al. ${ }^{23}$, but contrasted with the report of et al. ${ }^{22}$ on metabolic syndrome. Impairment of central insulin transport which is inversely associated with an increased energy intake and body weight with consequent decrease in insulin delivery to the central nervous system may be responsible for obesity and weight gain in MetS rats. ${ }^{25}$ Excessive fructose consumption has previously been reported to alter the serum concentrations of leptin and ghrelin leading to increased energy intake, weight gain and obesity. ${ }^{26}$

Supporting evidences on the relationship between serum lipids and metabolic syndrome continues to accumulate, the relation between dyslipidaemia and metabolic syndrome could also be conjectured. In this study, the significant increase in serum TC and LDL-C and decrease serum HDL-C concentrations in MetS rats corroborated with the report of previous studies, ${ }^{15,27}$ on metabolic syndrome. This study, however demonstrated no significant differences in the serum VLDL, TG and AIX between MetS group and control group. The observed dyslipidaemia could be due to exposure of the liver to 
an overload of fructose which in turn enhances glycogen storage directly from glucose or through gluconeogenesis. Unlike glucose, fructose bypasses the rate-limiting step catalysed by phosphofructokinase and stimulates fatty acid synthesis and hepatic triglycerol secretion ${ }^{28}$ and further lead to a chain of reactions in the lipoprotein and lipids leading to dyslipidaemia.

Metabolic syndrome is characterized by low-grade inflammatory state. In this study, the increased serum levels of IL- 6, IL-10 and TNF- $\alpha$ in fructose-induced MetS rats is in agreement with previous studies..$^{5-6,29}$ However, Matia-Garciaet al. ${ }^{30}$ and Srikanthan et al. ${ }^{31}$ reported no significant changes in the serum IL-10 levels between MetS state and in controls. Calcaterra et al..$^{32}$ in their study had earlier speculated that the increased IL-10 levels is an attempt by the body to inhibit the on-going proinflammatory cytokine production. However, Srikanthan et al. ${ }^{31}$ in their study reported no significant difference in serum TNF- $\alpha$ levels between MetS state and in controls. The increased serum TNF- $\alpha$ levels observed in this study may be a consequence of continual accumulation of lipids both in macrophages and adipocytes within the adipose tissues, leading to a shift in macrophage subtype to a pro-inflammatory M1 polarization..$^{33}$, with the resultant release of TNF- $\alpha$.

Interleukin- 6 has been implicated in inflammatory crisis and as a key player in the mediation of low-grade inflammatory process in tissues. ${ }^{34}$ In this study, increased levels of IL-6 was observed in MetS rats in comparison with the control group. Elevated serum levels of IL-6 have earlier been reported in many diseases including atherosclerosis, malnutrition, cardiovascular events and hypertension. ${ }^{34-35}$ It was suggested that, the secretion of IL-6 was closely related to obesity which is an important component of metabolic syndrome. ${ }^{34}$ and increased IL-6 levels in hypertensive patients was more marked among hypertensive adult patients with obesity. ${ }^{34-36}$

Attempt was also made to correlate the mean serum blood glucose and body mass index with the inflammatory cytokines in order to access the degree of the association between the two. The result in this study indicated that serum IL-10 and TNF- $\alpha$ were inversely correlated with BMI. Serum IL-10 also showed the same relationship with FBG while, serum TNF- $\alpha$ was not associated with FBG. Likewise serum IL-6 levels were not associated with either of BMI and FBG. The inverse correlation between IL-10 and TNF- $\alpha$ and BMI suggest that increased BMI resulted in the down regulation of the IL-10 and TNF$\alpha$ in fructose-induced MetS rats. Likewise, serum IL-10 negatively correlated with FBG which also transmitted the message that increase of FBG in MetS rats resulted in the down regulation of IL-10 in the serum of MetS rats. Serum TNF- $\alpha$ was not associated with FBG, and serum IL-6 levels were not associated with either of BMI and FBG. The positive correlation observed between serum TG and each of AIX and VLDL-C lead to increased atherogenic index of plasma and elevated levels of VLDL-C in MetS rats. With the exception of serum LDL-C which positively correlated with TC, significant correlation was not established between serum TC and each of AIX, HDL-C and VLDL-C in MetS rats. As expected, the inverse association between the levels of HDL-C and TG in MetS rats implies that a decreased in serum HDL-C resulted to significant elevation of triglyceride which contribute to dyslipidaemia in MetS rats.

\section{CONCLUSION}

In conclusion, the rat model of MetS was established after 32 weeks treatment with $10 \%$ fructose in water. This plays an important role in the pathogenesis of components of metabolic syndrome, including dyslipidaemia, hyperglycaemia and obesity; and serum IL-6, IL-10 and TNF- $\alpha$ are raised in MetS rats and this underscores the role of these cytokines in inflammation associated with metabolic syndrome.

\section{ACKNOWLEDGEMENT}

The authors wish to thank the management of School of Medical Laboratory Sciences and Animal House, Faculty of Pharmaceutical Sciences, Usmanu Danfodiyo University Sokoto for providing the enabling environment and the Laboratory facility.

\section{REFERENCES}

1. Yogita R, Naga VP, Swathi $K$ and Jawahar LM. Metabolicsyndrome: pathophysiology, management, and modulation by natural compounds. Ther Adv Cardiovasc Dis 2017; 11(8): 215-225.

https://doi.org/10.1177/1753944717711379

2. Eva K, Panagiota P, Gregory, K and George C. Metabolic syndrome: definitions and controversies. BMC Med 2011; $9(1): 48$.

https://doi.org/10.1186/1741-7015-9-48

3. Ha S, Choi HR and Lee YH. Clustering of four major lifestyle risk factors among Korean adults with metabolic syndrome. PLoS One2017; 12(3):e0174567. https://doi.org/10.1371/journal.pone.0174567

4. Lee WT, Lee CY, Tsai S, Huang HL, Wu PW, Chin Y, et al. Clustering of metabolic risk components and associated lifestyle factors: A nationwide adolescent study Taiwan. Nutrients 2019; 11(3): 584.

https://doi.org/10.3390/nu11030584 
5. Mirhafez SR, Pasdar A, Avan A, Esmaily H, Moezzi A, Mohebati M, et al. Cytokine and growth factor profiling in patients with the metabolic syndrome. Br J Nutr 2015; 113 (12):1911-1919. https://doi.org/10.1017/S0007114515001038

6. Schmidt FM, Weschenfelder J, Sander C, et al. Inflammatory Cytokines in General and Central Obesity and Modulating Effects of Physical Activity. PLoS One 2015; 10 (3): e0121971. https://doi.org/10.1371/journal.pone.0121971

7. Gregory JW. Prevention of obesity and metabolic syndrome in children. FrontEndocrinol 2019; 10: 669.

https://doi.org/10.3389/fendo.2019.00669

8. Bayturan O, Tuzcu EM, Lavoie A, Hu T, Wolski K, Schoenhagen $\mathrm{P}$, et al. The metabolic syndrome, its component risk factors, and progression of coronary atherosclerosis. Arch Intern Med2010;170(5): 478-484.

https://doi.org/10.1001/archinternmed.2009.551

9. Al-Gobban AS, Al-Alfi MA and Khan MZ. Mechanism linking diabetes mellitus and obesity. Diabetes Metab Syndr Obes 2014;7: 587-591.

https://doi.org/10.2147/DMSO.S67400

10. Harika RK, Eilander A, Assema M, Osendarp SJM and Zock PL. Intake of fatty acids in general populations worldwide does not meet dietary recommendations to prevent coronary heart disease: A systematic review of data from 40 countries. Ann Nutr Metab 2013;63: 229-238. https://doi.org/10.1159/000355437

11. Al-Bachir M. and Bakir MA. Predictive value of body mass index to metabolic syndrome risk factors in Syrian adolescents. J Med Case Rep 2017;11: 170. doi: 10.1186/s13256-017-1315-2. https://doi.org/10.1186/s13256-017-1315-2

12. Kaur JA. A Comprehensive review on metabolic syndrome. Cardiol Res Pract 2014; 21: 10-14. https://doi.org/10.1155/2014/943162

13. Pal $S$ and Ellis $V$. The chronic effects of whey proteins on blood pressure, vascular function, and inflammatory markers in overweight individuals. Obesity (Silver Spring) 2010;18: 1354-1359.

https://doi.org/10.1038/oby.2009.397

14. National Research Council (US) Committee for the Update of the Guide for the Care and Use of Laboratory Animals. Environment, housing and management. In: Guide for the care and use of laboratory animals ( $8^{\text {th }}$ edition). Washington, DC: National Academies Press 2011.

15. Toop CR and Gentili S. Fructose Beverage Consumption Induces a Metabolic Syndrome Phenotype in the Rat: A Systematic Review and Meta-Analysis. Nutrients 2016;8: 577. https://doi.org/10.3390/nu8090577

16. Ochei $\mathrm{J}$ and Kohatkar A. Medical Laboratory Science: Theory and Practice ( $1^{\text {st }}$ edn.). New Delhi: Tata McGraw-Hill. 2000; Pp101-102.

17. Nwagha UI, Ikekpeazu EJ, Ejezie FE, Neboh EE and Maduka IC. Atherogenic index of plasma as useful predictor of cardiovascular risk among postmenopausal women in Enugu, Nigeria. Afr Health Sci 2010; 10(3): 248-252.

18. Yener AU, SehitogluMH, Ozkan MTA, Bekler A, Ekin A and Cokkalender $\mathrm{O}$, et al. Effects of kefir on ischemia-reperfusion injury. Eur Rev Med PharmacolSci 2015; 19: 887-896.

19. Mustafa A, Ahmet V, Can K, Kemal O and Zeki A. Endothelial dysfunction in high fructose containing diet fed rats increased nitric oxide and decreased endothelin-1 levels in liver tissue. Dicle Medical Journal 2010;37(3): 193-198.

20. Maged AH, Laila AR and Marwa AM. The effect of high fat diet and high fructose intake on insulin resistance and GLP-1 in experimental animals. Med J Cairo Univ 2011;66(14): 1615-1624.

21. Ramadan KS, Yousef JM, Hamza AH and Abdel-Basset SE. A positive role for selenium in mitigating complications associated with fructose-induced metabolic syndrome in rats. Int $\mathrm{J}$ NutrMetab2012;4(11): 146-150.

22. Mohammed RS, Mehdi H and Ahmad RS. Prevention of high fructose induced metabolic syndrome in male Wistar rats by aqueous extract of Tamarindusindica seed. Acta Med Iran2010;49(5): 277-283.

23. Norshalizah M, Zar CT, Shaiful RS, Natasya NS, Mohd RM and Farihah HS. The establishment of metabolic syndrome model by induction of fructose drinking water in male Wistar rats. Biomed Res Int2014;20(14): 1-8. https://doi.org/10.1155/2014/263897

24. Sobczak IS, Blindauer CA and Stewart AJ. Changes in plasma free fatty acids associated with Type 2 diabetes. Nutrients 2019; 11(9): 2022. doi.org/10.3390/nu11092022. https://doi.org/10.3390/nu11092022

25. Dekker MJ, Su, Q, Baker C, Rutledge AC and Adeli K. Fructose: a highly lipogenic nutrient implicated in insulin resistance, hepatic steatosis, and the metabolic syndrome. Am J PhysiolEndocrinolMetab 2010; 299(5): 685-694. https://doi.org/10.1152/ajpendo.00283.2010

26. Johnson RJ, Nakagawa T, Sanchez-Lozada LG, Shafiu M, Sundaram S, Le M, et al. Sugar, uric acid, and the aetiology of diabetes and obesity.Diabetes 2013; 62(10): 3307-3315. https://doi.org/10.2337/db12-1814

27. Steckhan N, Hohmann CD, Kessler C, Dobos G, Michalsen A and Cramer $\mathrm{H}$. Effects of different dietary approaches on inflammatory markers in patients with metabolic syndrome: A systematic review and meta-analysis. Nutrition 2016; 32(3): 338. https://doi.org/10.1016/j.nut.2015.09.010

28. Fienman RD and Eugene JF. Fructose in perspective. NutrMetab 2013;10: 45. https://doi.org/10.1186/1743-7075-10-45

29. Udenze IC, Amadi EC, Awoola AN, Makwe CC and Ajie IO. Plasma Levels of inflammatory cytokines in adult Nigerians with the metabolic syndrome. Niger J Med2016;57(1): 64-68. https://doi.org/10.4103/0300-1652.180569

30. Matia-García I, Muñoz-Valle JF, Reyes-Castillo Z, GarcíaArellano S, Salgado-Bernabé AB, Del C Alarcón-Romero L, et al.Correlation between cytokine profile and metabolic abnormalities in young subjects. Int J Clin Exp Med2016;9(8): 16596-16604.

31. Srikanthan K, Feyh A, Visweshwar H, Shapiro JI and Sodhi K. Systematic Review of Metabolic Syndrome Biomarkers: A Panel for Early Detection, Management, and Risk Stratification in the West Virginian Population. Int J Med Sci 2016;13 (1): 25-38. https://doi.org/10.7150/ijms. 13800

32. Calcaterra V, De Amici M, Catherine K, Cristina T, Vincenza B, Francesca S, et al. Adiponectin, IL-10 and metabolic syndrome in obese children and adolescents. Acta Biomed2009; 80:117-123.

33. Prieur X, Mok CY, Velagapudi VR, Núñez V, Fuentes L, Montaner D, et al.Differential lipid partitioning between adipocytes and tissuemacrophages modulates macrophage lipotoxicity and M2/M1 polarization in obesemice. Diabetes2011;60:797-809. https://doi.org/10.2337/db10-0705

34. Bao P, Liu G and Wei Y. Association between IL-6 and related risk factors of metabolic syndrome and cardiovascular disease in young rats. Int J Clin Exp Med 2015; 8(8):13491-13499. www. ijcem.com /ISSN: 1940-5901/IJCEM0011888.

35. Bernberg E, Ulleryd MA, Johansson ME and Bergström GM. 
Social disruption stress increases IL-6 levels and accelerates atherosclerosis in ApoE-/-mice. Atherosclerosis 2012; 221: 359-365.

https://doi.org/10.1016/j.atherosclerosis.2011.11.041

36. Heresi GA, Aytekin M, Hammel JP, Wang S, Chatterjee S and
Dweik RA. Plasma interleukin-6 adds prognostic information in pulmonary arterial hypertension. Eur Respir J 2014; 43: 912-914.

https://doi.org/10.1183/09031936.00164713

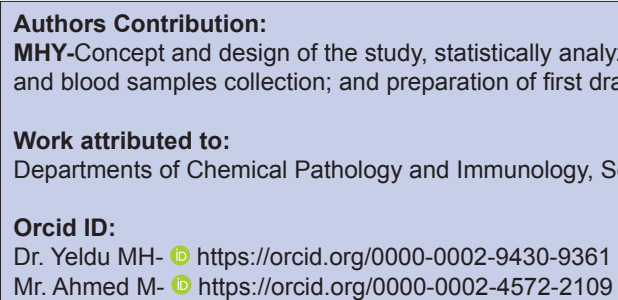

Work attributed to:

Orcid ID:

Dr. Yeldu MH- (i) https://orcid.org/0000-0002-9430-9361

Mr. Ahmed M- (D) https://orcid.org/0000-0002-4572-2109

MHY-Concept and design of the study, statistically analyzed, interpreted data and critical revision of the manuscript; AM-Reviewed the literature, data collection and blood samples collection; and preparation of first draft of the manuscript.

Departments of Chemical Pathology and Immunology, School of Medical Laboratory Sciences, Usmanu Danfodiyo University, Sokoto.

Source of support: None, Conflicts of Interest: None 